Man and Nature

MAN AND NATURE

L'homme et la nature

L'HOMME ET LA NATURE

\title{
Le principe de liberté et les contraintes institutionnelles envisagées dans les Cinq mémoires sur l'instruction publique de Condorcet
}

\section{Robert Niklaus}

Volume 10, 1991

URI : https://id.erudit.org/iderudit/1012630ar

DOI : https://doi.org/10.7202/1012630ar

Aller au sommaire du numéro

Éditeur(s)

Canadian Society for Eighteenth-Century Studies / Société canadienne d'étude du dix-huitième siècle

ISSN

0824-3298 (imprimé)

1927-8810 (numérique)

Découvrir la revue

Citer cet article

Niklaus, R. (1991). Le principe de liberté et les contraintes institutionnelles envisagées dans les Cinq mémoires sur l'instruction publique de Condorcet. Man and Nature / L'homme et la nature, 10, 133-146.

https://doi.org/10.7202/1012630ar

Copyright (c) Canadian Society for Eighteenth-Century Studies / Sociéte canadienne d'étude du dix-huitième siècle, 1991
Ce document est protégé par la loi sur le droit d'auteur. L'utilisation des services d'Érudit (y compris la reproduction) est assujettie à sa politique d'utilisation que vous pouvez consulter en ligne.

https://apropos.erudit.org/fr/usagers/politique-dutilisation/ 


\section{Le principe de liberté et les contraintes institutionnelles envisagées dans les Cinq mémoires sur l'instruction publique de Condorcet}

Tout nouveau programme d'éducation dérive d'une idée philosophique ou politique. Pour comprendre le projet de Condorcet il convient de préciser son intention et d'examiner les prémices sur lesquelles il se fonde. Lié avec d'Alembert qui a onze ans de plus que lui, qu'il a patronné et a avancé sa candidature à l'Académie des Sciences dont il devint le sécrétaire perpétuel, considéré comme un des premiers géomètres de l'Europe, Condorcet a contribué pour la partie mathématique au Supplément de l'Encyclopédie. Il partage avec Diderot, les philosophes du temps et les principaux collaborateurs de la grande entreprise du siècle les idées maîtresses que d'Alembert a mises en relief dans le Discours préliminaire, et il a certainement lu avec soin les articles de l'Encyclopédie portant sur l'éducation.

Son programme vise évidemment à consolider le triomphe des lumières, et les lycées qu'il voudrait instituer et que nous appelons aujourd'hui universités doivent être à ses yeux des 'encyclopédies vivantes.' Il y a plus. Il a compris que seule l'instruction peut rendre irréversible la grande révolution dans les sciences et les arts que les philosophes ont initiée. Les nouvelles découvertes doivent être mises à la portée de tous et un vaste programme d'éducation doit être mis en marche pour assurer le progrès indéfini de l'esprit humain, idée à laquelle il s'est accroché même dans la détresse de ses derniers jours. Son Esquisse d'un tableau historique des progrès de l'esprit humain a été critiquée, encore que l'optimisme qu' on lui reproche doive être qualifié, mais il faut à coup sûr reconnaître le mérite de sa foi qui anime toute son oeuvre et constitue un des grands ressorts de son action politique. De vingt-cinq ans le cadet de son ami Voltaire auquel il a rendu visite à Ferney en compagnie de d'Alembert et dont il a retracé la vie pour l'édition des Oeuvres de Voltaire dite de Kehl, Condorcet admire en lui les appels à la tolérance, l'homme qui a lutté contre les préjugés et l'oppression, les injustices de toute sorte. Il n'a jamais oublié l'homme 
de Calas, de Sirven, de La Barre et l'affaire Lally Tollendal dont la révision date de 1780 est présente à son esprit quand il prend position avec Dupaty dans l'affaire des trois roués. Toute injustice l'oppresse. Il est assoiffé d'équité et l'on peut dire que c'est son grand besoin de justice qui est à la base du principe d'égalité qu'il revendique. On a souvent dit que Condorcet raisonne en mathématicien; le mot 'donc' revient incessamment sous sa plume, et sa logique, objective et froide, entraîne la conviction. Il est aussi un cartésien dont la pensée déductive suit un développement linéaire. Il part de quelques axiomes très simples et très clairs, et par un enchaînement inévitable nous amène à une conclusion qui paraît l'évidence même. Il fait toujours appel au bon sens de chacun, à cette raison commune qui est l'apanage de l'individu et par delà lui de la collectivité. Sa manière de penser est à l'antipode de celle des esprits pragmatiques, ce que les hommes de son époque et les Anglais en particulier lui ont reproché. Il se méfie de l'histoire qui s'accroche au passé, car il croit qu'il faut nécessairement tout remettre en cause, tout repenser à neuf comme l'ont fait les grands philosophes du XVIIIe siècle. A cet égard Condorcet est par excellence l'homme de la révolution, mot qu'il faut prendre dans le sens de rupture avec le passé. C'est en effet avec 1789 que le mot est pris dans ce sens moderne - et par contraste la révolution anglaise de 1688, la 'Glorious Revolution' que certains historiens britanniques lui opposent, n'est somme toute qu'une révolution dans le sens copernicien du terme, enregistrant le triomphe du Parliament sur un roi qui voulait changer le régime, et malgré l'importance à accorder à l'Habeas Corpus de 1679 marque en réalité un retour au statu quo ante.

Condorcet est un penseur indépendant qui n'a pas le sens politique, c'est-à-dire l'esprit souple qui permet de concilier les intérêts contradictoires, de louvoyer quand les circonstances le demandent pour faire triompher son point de vue, de transiger. L'excellent ouvrage qu'Elizabeth et Robert Badinter viennent de lui consacrer a pour titre Un intellectuel en politique, ${ }^{1}$ titre qui a une résonnance très contemporaine et auquel on pourrait préférer Un philosophe en politique. Il indique cependant fort bien une caractéristique de l'esprit de Condorcet qui est la clé de son insuccès dans l'arène politique dans laquelle il est entré. Familier du salon de Mademoiselle de Lespinasse, en rapports étroits avec tant de personnalités de premier plan, avec Turgot et les physiocrates, La Fayette, La Rochefoucauld, Jefferson dont il fut l'ami, Thomas Paine qu'il fréquenta pendant deux ans, avec le monde des clubs révolutionnaires, avec les girondins en particulier, il ne fut d'aucun parti. Il n'accepta pas d'être franc-maçon comme Voltaire à la fin de sa vie. Au courant de tout, publiant avec un commentaire personnel qu'il conviendrait de rééditer, les procès verbaux de l'Assemblée, fondant des 
journaux assez éphémères, il veut agir sur l'opinion par la force de son argumentation. Mais il a beau présider de nombreuses séances de l'Assemblée Législative et des commissions auxquelles sa compétence reconnue, sa situation intellectuelle et sociale l'appelaient, il ne réussit à l'Assemblée que lorsque ses vues étaient precisément celles des députés, ce qui arriva très rarement. Sa voix grêle ne lui permettait pas l'éloquence d'un Mirabeau ou d'un Danton. On l'a appelé 'un mouton enragé,' ce qui est assez juste, mais sa rage était froide et ne se communiquait pas au grand public par la parole; 'un volcan couronné de neige,' disait d'Alembert; c' est son influence indirecte par ses interventions à la Chambre et surtout par ses écrits dans les journaux qui reste importante et mériterait une étude sérieuse.

Condorcet conçoit la politique comme une science, une machine qui fonctionne selon un calcul de probabilités. Il a fait l'apologie des lois et a sans doute accordé une confiance excessive à la législation. Selon lui l'État subsiste grâce à son pouvoir législatif, mais, tout en reconnaissant la diversité des facteurs qui doit déterminer les lois, il veut maintenir des critères absolus. Il croit fermement en une justice universelle qui émane de la raison pure, même si celle-ci repose sur un calcul de probabilités et non sur un principe divin. Il s'écarte nettement de Montesquieu pour lequel les lois sont les rapports nécessaires qui dérivent de la nature des choses, par conséquent relatives et sujettes à des nécessités politiques, sociales, économiques, psychologiques, voire climatologiques. Si Montesquieu trouve des raisons qui expliquent les lois, ces dernières ne sont pas pour autant rationnelles. Pour Condorcet les lois doivent dériver de principes. Il a commenté Montesquieu avec une certaine hargne comme l'a relevé Paul Vernière qui pense par ailleurs que Montesquieu est concordataire plus que révolutionnaire. Condorcet aussi en ce qu'il tient compte de la totalité des citoyens, d'un consensus qui doit respecter la liberté individuelle. ${ }^{2}$ A première vue il semble plus proche de Rousseau qui a conçu un Législateur idéal qui interprête la volonté générale; mais celle-ci, hélas, n'est pas toujours éclairée et il craint que la souveraineté du peuple n'aboutisse à un despotisme aussi néfaste que celui des rois. Robespierre lui a reproché son jurisme qui a, cependant, le mérite d'être à la base de la Constitution et des Droits de l'Homme, antérieurs selon lui à la société ellemême, qu'il a préconisés pour défendre la liberté individuelle que l'éducation doit promouvoir tout en diminuant l'inégalité. Pour le Britannique la liberté consiste en la faculté de faire tout ce que la loi ne défend pas. Aux yeux de Condorcet cela est bien insuffisant pour protéger les femmes, les noirs, les protestants et les juifs auxquels il voudrait donner le droit de cité. Dans son sytème même les étrangers auraient le droit de vote; mais ce droit de vote ne peut garantir en soi 
la protection efficace des minorités. Il condamne le système parlementaire anglais avec ses deux chambres qui ne représentent pas vraiment la volonté du peuple et il réclame un processus électoral avec représentation proportionnelle dont il a déterminé les modalités avec une précision mathématique. L'avantage de son système n'est pas seulement d'être plus juste, mais aussi d'enrayer l'établissement de fortes majorités qui donnent trop de pouvoir à l'État. Il ferait volontiers place au referendum et à la possibilité d'une initiative populaire. Il entrevoit que la souveraineté de l'assemblée peut entraîner le despotisme du chef de l'État, même si en dernière analyse celui-ci n'exerce le pouvoir que grâce à la passivité des sujets, des désavantagés qui conservent le droit de résister à l'oppression. Il faut donc former un citoyen actif et indépendant et envisager des réformes constitutionnelles permettant des coalitions qui concilient les divers intérêts. La Déclaration des droits de l'homme, et par là il faut évidemment inclure les droits de la femme, est à ses yeux la grande contrainte institutionnelle qui limite le pouvoir de l'État. On a relevé que ce texte ne spécifie pas le droit à l'éducation, mais en fait celui-ci est implicite dans ses écrits. Relevons encore qu'il ne s'agit pas des droits des hommes en général, mais de l'homme au singulier; c'est dire que dans l'opposition individu et collectivités Condorcet opine sans hésiter pour l'individu. Il réclame la liberté des personnes et des biens, l'égalité devant la loi, la loi fiscale y compris, la révision de tout le système juridique, et suit Beccaria pour les délits et les peines. Il recherche le vote unanime des jurés qui seraient tirés au sort. Il n'accepte ni lettres de cachet ni corvées et veut la liberté de la presse. Il voudrait fonder une Trésorerie Nationale et un Bureau de Comptabilité indépendant du Conseil exécutif dont du reste les membres seraient élus. Les ministres non plus ne seraient pas désignés par le chef de l'État, mais élus. La présidence de l'Assemblée serait tournante pour éviter le danger d'un monarque républicain, et l'on conserverait le droit de faire réexaminer les lois après vingt ans. Il craint donc que la souveraineté du peuple soit confisquée ou altérée par ses représentants et tient absolument à limiter les pouvoirs. Aussi les lois doivent-elles protéger l'école contre les pouvoirs, et ce faisant renforcer les libertés inscrites dans la Déclaration des droits de l'homme. Il condamne les parlements qui ont jadis approuvé les massacres de la Saint-Barthélémy et dont il a reconnu le despotisme. La question de l'éducation est pour Condorcet décisive. C'est pourquoi il lui a consacré un des plus longs rapports qu'il ait rédigés et certainement le plus substantiel qui ait été publié durant et après la Révolution. Les Cinq Mémoires sur l'instruction publique parurent en 1791 dans la Bibliothèque de l'Homme public qu'il avait fondé avant même la proclamation de la République en septembre 1792, ce qui témoigne de 
l'importance qu'il attache à l'éducation. S'il cherche à former le citoyen de la république qui s'annonce, ce citoyen est avant tout un homme qu'il s'agit d'instruire pour qu'il soit libre et puisse participer dans un esprit d'indépendance à la vie sociale. Ici, comme l'a bien vu Charles Coutel dans l'édition des Cinq Mémoires qu'il vient de publier avec Catherine Kintzler, ${ }^{3}$ Condorcet fait preuve d'originalité. Contrairement aux révolutionnaires de 1789 , il s'oppose au modèle spartiate, au système éducatif de Lycurgue repris par Plutarque, Montaigne et Rousseau, selon lequel l'on peut penser à la fois l'école et la cité politique comme une seule et même idée. Pour Charles Coutel que nous citons avec quelques coupures et auquel nous renvoyons pour une plus ample information le modèle lacédémonien recèle six points que Condorcet ne saurait accepter:

1. Une hérö̈sation vertueuse du philosophe-législateur, fondateur d'un ordre politique durable et unanime.

2. Une décentralisation généralisée dans le temps et dans l'espace. Le spartiate ne fait pas autre chose quand il déclare dans les Confessions: 'Je me croyais Grec ou Romain!'.

3. Une collectivisation de la vertu morale. Le modèle spartiate d'une société unanime et vertueuse se manifeste par un bellicisme qui aboutit à la proscription des commerces et des arts.

4. Une réduction de la citoyenneté à la nationalité, ce que La Chalotais et Ducos avaient entrepris dans leur lutte contre les jésuites ultramontains et les cléricaux. Comme le souligne Ch. Coutel: 'C'était ne pas voir qu'à un cléricalisme religieux on risquait de substituer un cléricalisme politique et civique, potentiellement belliciste comme fut le cas en 1792-94.'

5. Un sacrifice de l'individu au profit de la patrie. Cette désindividualisation entraîne une démocratie d'association et d'appartenance au détriment $d$ 'une république fondée sur les garanties écrites des libertés individuelles et publiques.

6. Enfin et surtout une dissolution du problème de l'école. La référence à Sparte permettait de se dispenser de réfléchir sur l'acte d'instruction et sur l'école en général. Dans le modèle lacédemonien, l'école est pur effet de la volonté sociale et politique. Ce culte spartiate fut exprimé par Saint-Just et Robespierre et dans le domaine de l'école par Le Peletier de Saint Fargeau, Rabaut Saint- 
Etienne et Ducos, pour lesquels la société vertueuse et unanime doit trouver son reflet dans l'École. Les enfants doivent être élevés pour s'intégrer dans la société du jour jugée parfaite. Condorcet pour sa part se rallie à la pensée de Voltaire, de Diderot et surtout de Turgot quand il écrit dans le Premier Mémoire qu'en sacrifiant l'individu Sparte a rendu impossible l'exercice de la raison. Les tenants du 'fantasme spartiate' pour employer l'expression de Ch. Coutel, mettent en scène l'essor des lumières, de la raison et en définitive de la Révolution elle-même. 'A la fin de sa vie, dans les Fragments sur l'Atlantide, Condorcet opposera à Sparte modèle obscurantiste, régressif, conservateur et clérical, le mythe régulateur, progressiste et mobilisateur de l'Atlantide.' Il dit encore: 'Cette réunion de tous les hommes qui dans une même nation font du soin de cultiver leur raison, d'augmenter leurs lumières, ou leur occupation, ou leur plaisir, peut s'étendre à toutes les nations éclairées. ${ }^{\prime 4} \mathrm{Il}$ s'agit donc d'instruire les enfants pour qu'ils puissent nous critiquer et non pas nous ressembler, et ne pas les conditionner à un destin collectif. Condorcet rejette donc un simpliste critère d'utilité publique, surtout celui d'utilité immédiate prescrit par Helvétius.

L'éducation de base doit être générale; l'école doit communiquer les connaissances nécessaires à tout homme ou femme, laissant à plus tard le soin de préparer aux diverses vocations et métiers, les orientations dont il voudrait cependant accélérer le cours. 'La division des métiers,' dit-il, 'si elle se fait trop tôt, doit conduire le peuple à la stupidité.' L'instruction donnée magistralement doit se borner à familiariser les élèves avec les faits avérés, et doit être neutre. C'est de propos délibéré qu'il adopte le mot 'instruction' qu'il préfère à 'éducation,' celle-ci devant se poursuivre durant toute la vie et étant le résultat d'expériences multiples à commencer par celles acquises au sein de la famille et par l'observation. Tout son système repose sur le principe d'égalité comme l'a bien vu K.M. Baker ${ }^{6}$ dans les excellentes pages d'analyse qu'il a rédigées sur le projet de Condorcet. En effet Condorcet insiste dès le début sur ce principe d'égalité qui dérive de la notion d'équité selon laquelle chacun doit pouvoir jouir des mêmes droits.

'Le maintien de la liberté et de l'égalité,' dit-il, 'exige un certain rapport entre l'instruction des citoyens qui en peuvent recevoir le moins et les lumières des hommes les plus éclairés dans le même pays et à la même époque. Il exige également une certaine proportion entre les connaissances des hommes et leurs besoins. ${ }^{7}$ Observation qui reste pleinement valable aujourd'hui. 
S'il songe à une distribution plus équitable des fortunes ou plutôt des jouissances, il n'est aucunement en faveur de l'égalitarisme. Ce qu'il recherche, c'est l'égalité devant la loi - ce qui, entre parenthèses, l'amène à vouloir qu'il n'y ait pas une trop grande disproportion entre la condition de l'inculpé et de son juge - l'égalité des droits se complétant par l'égalité dans les chances de réaliser son potentiel. Mais Condorcet n'a jamais pensé que les inégalites dérivent simplement de différences dans l'éducation et l'ambiance. L'État a surtout le devoir d'offrir au citoyen la possibilité de développer ses talents. K.M. Baker oppose Condorcet à Jefferson qui, lui, place clairement la liberté individuelle en premier. ${ }^{8}$ Mais en fait si Condorcet insiste sur la nécessité de l'égalité, c'est que celle-ci est la sauvegarde de la liberté individuelle. 'Si une égalité réelle ne s'unit pas à l'égalité politique, alors le but de la société n'est pas rempli, ${ }^{\prime 9}$ dit-il. Madame Kintzler a très bien observé que sans égalité il n'y a pas de liberté. C'est au nom de l'égalité des droits qu'il revendique une éducation commune pour les garçons et les filles avec des instituteurs des deux sexes, pour tous, quels que soient la religion ou la couleur de la peau, le degré de richesse ou de pauvrete d'un particulier quelconque. Citons à ce propos un passage capital sur l'enseignement à donner aux filles et aux garçons qui n'a été pleinement apprecié qu'au vingtième siècle:

Nous avons prouvé que l'éducation publique devait se borner à l'instruction: nous avons montré qu'il fallait en établir les différents degrés. Ainsi, rien ne peut empêcher qu'elle ne soit la même pour les femmes et pour les hommes. En effet toute instruction se bornant à exposer des vérités, à en développer les preuves, on ne voit pas comment la différence des sexes en exigerait une dans le choix de ces vérités, ou dans la manière de les prouver. Si le système complet de l'instruction commune, de celle qui a pour but d'enseigner aux individus de l'espèce humaine ce qu'il leur est nécessaire de savoir pour jouir de leurs droits et pour remplir leurs devoirs, paraît trop étendu pour les femmes qui ne sont appelées à aucune fonction publique, on peut se restreindre à leur faire parcourir les premiers degrés, mais sans interdire les autres à celles qui auraient des dispositions plus heureuses, et en qui leur famille voudrait les cultiver. ${ }^{10}$

Comme pour La Chalotais l'éducation pour Talleyrand doit être conçue essentiellement pour le plus grand bien de l'État. Talleyrand, qu'on a appelé le pédagogue de la Constituante, est à coup sûr un politicien très habile, mais son raisonnement spécieux qui a prévalu pendant longtemps ne fait en réalité que renforcer un préjugé. 
Une moitié du genre humain (les femmes), dit-il, exclue par l'autre de toute participation au gouvernement; des personnes indigènes par le fait et étrangères par la loi sur le sol qui les a cependant vu naître; des propriétaires sans influence directe et sans représentation; ce sont là des phénomènes politiques qu'en principe abstrait il paraît impossible d'expliquer; mais il est un ordre d'idées dans lequel la question change et peut se résoudre facilement. Le but de toutes les institutions doit êtrele bonheur du plus grand nombre. Tout ce qui s'en écarte est une erreur; tout ce qui y conduit une vérité. Si l'exclusion des emplois publics prononcée contre les femmes est pour les deux sexes un moyen d'augmenter la somme de leur bonheur mutuel, $c^{\prime}$ est dès lors une loi que toutes les sociétés ont dû reconnaitre et consacrer. ${ }^{11}$

Le dix-neuvième siècle qui a idéalisé la femme l'a aussi confinée dans son rôle d'épouse et de mère.

Cette instruction nationale essentielle ne sera pourtant pas absolument obligatoire, car on ne doit pas forcer à apprendre ceux qui refusent de s'instruire, encore que Condorcet n'envisage guère qu'un grand nombre de parents refuse gratuitement l'occasion de développer l'intelligence de leurs enfants. Elle sera de nécessité laïque. Certains critiques ont pensé que l'athéisme et la haine du clergé ont poussé Condorcet dans ce sens. En réalité il n'a jamais parlé de supprimer les écoles catholiques, il a même vu dans leur existence une concurrence utile au maintien du niveau des études; mais il n'a pas consenti que ces écoles religieuses reçoivent la moindre subvention de l'État, les bibliothèques, cabinets et laboratoires devant être pourvus dans les seules institutions nationales. Par ailleurs il s'oppose vigoureusement aux congrégations religieuses, aux corps perpétuels tels les Oratoriens, et avec quelque flottement aux guildes et corporations de toute espèce. Tout en reconnaissant l'utilité des associations professionnelles, il refuse toute servitude professionnelle et ne veut pas d'équipes pédagogiques, parce qu'elles risquent de porter atteinte aux libertés individuelles.

La question de la gratuité de l'enseignement n'est pas abordée dans les Mémoires comme elle l'a été dans le Rapport et projet de décret sur l'organisation générale de l'instruction publique que Condorcet soumit à l'Assemblée Législative les 20 et 21 avril 1792 en tant que rapporteur du Comité d'instruction publique dont il fut élu président le 30 octobre 1791 et encore en 1792. Celui-ci découle, lui aussi, du principe d'égalité et reprend les idées des Cinq Mémoires, tout en précisant son plan d'études, orienté vers les sciences physiques et morales. Les trois stages prévus dans les Mémoires sont portés à cinq: primaire de 5 à 10 ans, secondaire de 10 à 13, etc. On y indique les écoles à créer (une école pour 4000 habitants), les instituts (nos lycées) au nombre de 110, les lycées (nos 
universités) au nombre de neuf, et la Société Nationale de savants qui est appelée à couronner tout l'édifice. Condorcet précise la durée des cours, les matières à enseigner et les méthodes à suivre. Il exprime certaines réserves sur l'histoire. Voulant rompre avec le passé, il veut une histoire moderne qui présenterait les droits de l'homme et traiterait des inégalités sociales et du progrès humain. Il insiste sur une formation professionnelle, mais sous-estime les difficultés de recrutement initiales, croyant pouvoir résoudre le problème par la nomination d'instituteurs dépourvus de grades travaillant sous la direction d'enseignants qualifiés. Il veut des écoles normales et demande, en sus de bâtiments, des bibliothèques et des laboratoires, des cabinets de machines et $\mathrm{d}$ 'histoire naturelle, des manuels et des dictionnaires, des journaux et des almanachs. L'éducation devant se poursuivre durant toute la vie, il veut encore instituer des cours publics pour adultes comme complément d'instruction, tels ceux qu'il avait lui-même inaugurés en 1786, destinés à libérer l'esprit humain. La vulgarisation des connaissances forme une partie essentielle de son programme, parce qu'elle permet l'exposition des principes, préface nécessaire à l'étude des sciences elles-mêmes. $C^{\prime}$ est dans cet esprit que dans les derniers jours de sa vie il a lui-même rédigé un traité de mathématiques élémentaires destiné à sa fille. ${ }^{12} \mathrm{Il}$ veut former des citoyens dignes de leur liberté, capables de progresser intellectuellement grâce à leur esprit critique. On peut parler si l'on veut de propagande philosophique, mais non pas de propagande politique entraînant un asservissement intellectuel.

Nous voici maintenant au coeur du problème pour Condorcet. D'une part il veut que l'Assemblée nationale élue démocratiquement et représentant les voix du peuple gouverne et donne l'initiative dans tous les domaines, mais de l'autre il tient à défendre les droits de l'individu. L'Assemblée a le devoir de formuler des lois pour le plus grand bien de l'individu aussi bien que de la société, d'enregistrer les résultats des progrès scientifiques, technologiques et autres, et le député dans l'intérêt de tous doit se laisser guider par la raison sans demeurer le simple porte-parole de ceux qui l'ont élu. En dernière analyse la raison et la vérité, en un mot les lumieres, doivent triompher de l'erreur, de la superstition, du fanatisme et des préjugés. La bonne politique se déduit par la raison des principes du droit naturel. A vrai dire Condorcet accorde une confiance extraordinaire à la législation. Celle-ci, cependant, doit être soumise aux droits de l'homme, partie intégrante de la constitution. Son expérience politique comme membre de la Législative, puis de la Convention ainsi que les aspirations des montagnards avant même l'avènement de Robespierre l'inquiètent. Il sait que la réforme de l'enseignement ne peut être réalisée que par un décret, que le gouvernement central devra fonder et financer les institutions nouvelles, que les 
enseignants devront être stipendiés par l'État qui devra par ailleurs déterminer les nouveaux programmes et assurer l'agencement et le succès de la grande révolution pédagogique qu'il projette. Mais comment alors réconcilier le rôle directeur du gouvernement avec l'indépendance de l'école? Sa réponse, intéressante, prête à discussion. Pour empêcher la main-mise de l'État sur le processus éducatif il réclame l'indépendance des maîtres, de leurs organisations, et leur laisse le choix des méthodes à adopter, tout en soulignant la nécessité d'inspecteurs. Il enlève à 1 État le droit de nommer les instituteurs et professeurs dans les trois niveaux d'enseignement envisagés dans les Mémoires. Les instituteurs du primaire seront élus par les enseignants du secondaire et ceux-ci par les enseignants du niveau supérieur; les professeurs des lycées devront nommer ceux des instituts. Mais chaque municipalité aurait le droit de réduire la liste des éligibles. La Société nationale des savants qui doit avoir la fonction de surveiller l'éducation publique et de déterminer les travaux de recherches dans toutes les branches devra se recruter par un système de cooptation et peut inclure des étrangers. Si cette Société qui a encore pour tâche de s'occuper de la formation des enseignants et de rester en rapport avec les sociétés savantes à l'étranger comme en France, doit être financée par l'État, ses membres ne seront pas nommés par lui et restent souverainement indépendants. Son rôle devait dépasser celui de toutes les Académies dont Condorcet avait fait partie; mais c'était faire grande confiance aux spécialistes dans chaque matière et à leur compétence sociale et politique. Et l'Institut de France qu'il a largement contribué à fonder n'a jamais disposé de crédits aussi substantiels, ni de pouvoirs aussi étendus que ceux qu'il avait envisagés. L'on voit dans quelle mesure le système d'éducation de Condorcet est élitiste, du moins après le niveau du secondaire. La palme est accordée aux plus doués, aux plus intelligents. Si chacun a sa chance, l'intellectuel, le savant qui gagne dans les concours et qui mérite la confiance et l'appui de ses supérieurs ou de ses pairs doit jouir de la considération générale et remplir les plus hautes charges de l'État. A cet égard la France par ses grandes écoles a certainement favorisé les intellectuels bien plus que l'Angleterre avec ses 'public schools.' Condorcet a vraiment préconisé une méritocratie.

Une contrainte d'ordre général peut encore s'exercer sur les représentants du peuple. C'est 'l'arithmétique sociale' ou plutôt 'la mathématique sociale,' terme plus compréhensif auquel Condorcet a donné la préférence. Cette mathématique conçue par Sir William Petty en 1691 passe à juste titre pour avoir été fondée par Condorcet. Il a compris très vite qu' elle allait non seulement servir à désacraliser les sciences morales mais encore à jouer un grand rôle politique. Elle dépasse de beaucoup la statistique annuelle dont Gournay et Necker avaient senti le besoin 
ainsi que la statistique officielle et neutre telle que Peuchet l'a demandée en 1803. Dans son important ouvrage intitulé Essai sur l'application de l'analyse à la probabilité des décisions rendues à la pluralité des voix publié en 1785 et que Fiona Sommerlad et Iain McLean viennent de traduire en anglais, ${ }^{13}$ et qui est par endroits de lecture difficile, Condorcet se lance dans un calcul de probabilités où le hasard doit jouer un rôle négligeable et qui fonde son système de votation, mais encore le principe des sondages qui permettent de calculer les réactions du public grâce aux réponses faites par un petit nombre d'individus à des questions précises. Condorcet examine le problème et explicitement en termes probabilistes celui du passage de l'individuel au collectif que soulève un pareil exercice. Il en arrive à opposer les variabilités particulières et l'uniformité de l'agrégat qui les rassemble, créant ainsi ce qu'on a appelé sans doute à tort le 'paradoxe de Condorcet' qu'Oleg Arkhipoff, administrateur de l'Institut National de la Statistique et des Etudes Economiques de Paris, vient d'élucider. ${ }^{14}$ Selon celui-ci la grande découverte de Condorcet n'est pas d'avoir indiqué les difficultés du transfert des votes dans un système de scrutin visant à représenter exactement la volonté de la majorité ni d'avoir compris qu'une moyenne n'a de sens que lorsqu'il y a possibilité d'accord entre les termes diamètralement opposés, mais d'avoir vu que le passage d'un niveau d'agrégation à un autre s'accompagne de phénomènes logiques et que tout niveau d'agrégation constitue un univers sémantique autonome. Il suffit ici de relever que si le représentant du peuple doit pouvoir tenir compte d'une information fondée sur des statistiques, il reste toujours une part d'approximation dans cet exercice. Le représentant du peuple conserve donc sa liberté et en dernière analyse doit se laisser gouverner par sa raison, car, comme l'a dit Condorcet, 'La puissance publique n'est autre chose que l'organe de la raison commune. ${ }^{15} \mathrm{La}$ mathématique sociale est psychologiquement utile, surtout quand elle peut alerter un gouvernement solidement incrusté sur les dangers d'une politique doctrinaire qui n'est pas acceptable à la majorité.

Il est une contrainte, évidente à nos yeux, que Condorcet, ancien Inspecteur des Monnaies et ami de Turgot, n'a pu ignorer, mais qu'il a refusé de considérer: celle des fonds disponibles. Si la main-mise sur les biens du clergé et des émigrés ne pouvait suffire à faire les frais de son programme, il a pu songer, en sus d'impôts, à des souscriptions particulières pour augmenter les fonds nationaux. Mais dans les Cinq Mémoires il n'est rien dit sur cette question pourtant capitale. Dans le Projet de décret il a tout uniment chargé M.Romme du problème financier. Celui-ci s'est borné à réclamer la priorité pour le projet en insistant sur le fait que les études ne sont pas une charge mais un soulagemnent réel pour le citoyen. Il ajoute en note: 
Il est bon de remarquer que l'instruction ne consumera pas la dix-huitième partie de l'impôt total, qu'elle coûtera six fois moins que ne coûtaient les dépenses de l'ancien culte, et moins du tiers de ce que coûte le nouveau. ${ }^{16}$

En pratique les lourdes charges nouvelles et les frais de l'entretien d'une guerre onéreuse ont entraîné le renvoi de l'enseignement gratuit à tous les stages à une date ultérieure qu'on s'est gardé de préciser. Mais pour Condorcet la question budgétaire est sans intérêt car l'État a le devoir de faire suite à son projet d'instruction publique quel qu'en soit le coût.

En conclusion la réforme de l'enseignement de Condorcet se marque par un échec certain. Le Directoire a mis en cause l'instruction publique gratuite et Napoléon a abandonné le primaire à l'Eglise pour affermir son régime. Il a fallu attendre Jules Ferry et les lois de 1882-83 pour consolider le primaire. Des considérations de toute espèce et le plus souvent peu louables ont empêché la réalisation de son programme. Condorcet a souffert de n'avoir pas su estimer juste les volontés divergentes de ses collègues et les résistances de la majorité. Il n'a su ni transiger ni temporiser. $S^{\prime} i l$ a donné le branle à certaines innovations importantes, ses réformes d'ordre pratique ont été assez vite dépasssées. Ce qui reste cependant et intéresse aujourd'hui ce sont les grands principes généraux qui l'animent et la force du raisonnement qui les appuie. Il est de lui comme des grands philosophes du temps; leur pensée s'étend au-delà de l'actualité et a une perennité qu'ignorent les esprits pragmatiques attachés au seul présent. Certes ses mémoires pédagogiques n'ont pas l'ampleur, la profondeur et le style de l'Émile. Francisque Vial dans une étude assez ancienne ${ }^{17}$ a cru pouvoir montrer une filiation entre Condorcet et Rousseau, alors que Mme Kintzler et $\mathrm{Ch}$. Coutel voient une opposition irréconciliable entre eux. Il est vrai que les points de ressemblance demeurent superficiels, que le système selon lequel un précepteur peut se subordonner complètement à un élève est nécessairement différent $d$ 'un système $d$ 'instruction publique, que les principes de base sont différents et que l'éducation de Sophie part d'une conception du rôle de la femme dans la société que Condorcet ne peut accepter. Surtout leurs théories politiques et sociales s'opposent radicalement. Condorcet pour sa part reste un individualiste libéral qui voudrait établir un équilibre entre le bonheur de l'individu et l'intérêt de la société. Il repousse l'étatisme pur et se fait l'apôtre d'un libéralisme collectif qu'il a du mal a préciser; mais le problème qu'il a soulevé est encore le grand problème pour nos démocraties modernes, celui des rapports entre l'État et l'école, entre un gouvernement centralisé et $l^{\prime}$ individu. Ses solutions, il faut le reconnaître, $n$ 'offrent pas de garanties suffisantes. Comment assurer que l'État qui détient les fonds n'intervienne pas dans les affaires qui touchent à l'éducation? L'État n'est-il pas 
en droit de réprimer les révoltes et les émeutes? Peut-on lui enlever le contrôle politique? D'autre part le savant reste-il toujours d'une objectivité politique irréprochable? L'instituteur et le professeur ne demeurent-ils pas toujours en mesure d'endoctriner leurs élèves? Et ceux-ci une fois éduqués et libres seront-ils nécessairement des êtres moraux et 'vertueux' comme on disait alors, les appuis sûrs d'un régime démocratique républicain? Les individus ne restent-ils pas souvent égoïstes et ne considèrent-ils pas souvent la communauté comme un cadre pour leurs ambitions personnelles? La liberté et l'égalité entraînent-elles nécessairement la fraternité? Suffit-il enfin de combattre l'ignorance pour assurer le triomphe du droit et de la justice? Ce sont là autant de questions auxquelles les réponses de Condorcet peuvent sembler quelque peu naïves. Mais il nous incombe de respecter un idéalisme tenace, une confiance en la perfectibilité de l'homme qui est la condition de tout progrès social. ${ }^{18}$

\section{ROBERT NIKLAUS \\ University of Exeter}

\section{NOTES}

1 Elizabeth et Robert Badinter, Condorcet. Un intellectuel en politique (Fayard, 1988).

2 Paul Vernière, Montesquieu et l'Esprit des lois ou la raison impure (Sedes, 1977), pp.8, 136.

3 Condorcet, Écrits sur l'instruction publique. Volume Premier. Cing Mémoires sur l'instruction publique. Texte présenté, annoté et commenté par Charles Coutel et Catherine Kintzler (Les Classiques de la République, Edilig, 1989). Le second volume présenté par Charles Coutel donne le texte du Rapport sur l'instruction publique. Nous renvoyons aux pages 21-31 du volume premier pour le passage de Charles Coutel que nous citons en abrégeant par endroits. Sur la formation du citoyen, voir C. Kintzler, pp. 9-20, et son ouvrage Condorcet: L'instruction publique et la naissance du citoyen (Gallimard, 1987).

4 Voir ouvr. cité, p. 29.

5 Voir Oeuvres de Condorcet, ed. A. Condorcet \& O'Connor \& M.F. Arago (Paris, Firmin-Didot frères, 1847), t. VII, p. 192.

6 Keith Michael Baker, Condorcet. From Natural Philosophy to Social Mathematics (Chicago and London: The University of Chicago Press, 1975), pp. 293-303. Voir aussi la note thématique fort utile de Mme Kintzler dans l'édition des Cinq Mémoires citée, pp. 249-52.

7 Oeuvres de Condorcet, éd. citée, VII, pp. 440-41.

8 Voir aussi Elaine McAllister, 'Condorcet and Jefferson on Education,' Condorcet Studies, II, pp. 106-109. 
9 Oeuvres de Condorcet, éd. citée, VII, p. 389.

10 Ibid., VII, pp. 215-216. Sur l'éducation des filles au XVIIIe siècle, voir R. Chartier, M. Compère, D. Julia, L'éducation en France du XVIe au XVIIIe siècle (Paris, SEDES, 1976), p. 208.

11 Voir P. Chevallier et B. Grosperrin, L'enseignement français de la Révolution à nos jours. II. Documents. Paris et La Haye, Mouton, 1971, qui donne aux pages 1-10 des extraits du Rapport de Talleyrand ainsi que du Discours sur l'Éducation Nationale de Mirabeau.

12 Voir Condorcet. Moyens d'apprendre à compter sûrement et avec facilité, éd G. Schubring, N. Picard, C. Coutel. Editions Art, Culture, Lecture (Paris, 1989). Réimpression du texte publié par Sophie de Grouchy en 1799, avec un important appareil critique.

13 In The Political Theory of Condorcet (Social Studies Centre, George Street, Oxford).

14 Voir Actes du Colloque Condorcet (Paris, 8-11 juin, 1988), 'Le Paradoxe de Condorcet est-il un mythe?', pp. 92-100 in Condorcet mathématicien, économiste, philosophe, homme politique, éd. P. Crépel et C. Gilain (Minerve, 1989) et sa communication au Colloque La Pensée économique pendant la révolution (Musée de la Révolution française, Vizille, 6-8 septembre 1989) intitulée 'Une révolution dans la Révolution: Condorcet, Lavoisier et Peuchet.'

15 Oeuvres de Condorcet, éd. citée, VII, p. 372.

16 Oeuvres de Condorcet, VII, p. 571.

17 La doctrine d'éducation de Jean-Jacques Rousseau (Paris, Delagrave, 1920), pp. 188-204.

18 Il est vrai que cette confiance dans le progrès de l'esprit humain est moins absolu qu'on ne l'a cru et se ramènerait en définitive à l'espoir d'un monde meilleur, espoir qu'il partage avec Jefferson. Voir Renée Waldinger, 'Condorcet: The problematic nature of progress,' Condorcet Studies, I, pp. 117-129. 